\title{
Monocyte function-associated antigen expression during and after pediatric cardiac surgery
}

\author{
Peter Gessler, MD, ${ }^{\text {a }}$ Rene Pretre, MD, ${ }^{\mathrm{b}}$ Christoph Bürki, MD, ${ }^{\mathrm{c}}$ Valentin Rousson, ${ }^{\mathrm{e}}$ Bernhard Frey, MD, ${ }^{\mathrm{a}}$ and \\ David Nadal, MD ${ }^{d}$
}

Extra illustrations are available online. $\widetilde{\beta}$

\footnotetext{
From the Divisions of Pediatric Intensive Care Medicine, ${ }^{\text {a }}$ Cardiovascular Surgery, ${ }^{\mathrm{b}}$ Anesthesiology, ${ }^{\mathrm{c}}$ and Infectious Diseases, ${ }^{\mathrm{d}}$ University Children's Hospital of Zurich, Zurich, Switzerland, and the Department of Biostatistics, ${ }^{\mathrm{e}}$ University of Zurich, Zurich, Switzerland.

There is no conflict of interest.

The study was financially supported by a grant from EMDO Stiftung, Zurich, Switzerland.

Received for publication Nov 4, 2004; revisions received Dec 17, 2004; accepted for publication Jan 10, 2005.

Address for reprints: Peter Gessler, MD, University Children's Hospital, Steinwiesstr 75, CH 8032 Zurich, Switzerland (E-mail: peter.gessler@kispi.unizh.ch)

J Thorac Cardiovasc Surg 2005;130:54-60

$0022-5223 / \$ 30.00$

Copyright () 2005 by The American Association for Thoracic Surgery

doi:10.1016/j.jtcvs.2005.01.008
}

Objective: Systemic inflammatory response syndrome and infectious complications are major causes of morbidity and mortality after cardiopulmonary bypass. Recent work in adult patients suggests that the balance between proinflammatory and anti-inflammatory mediators is important. We hypothesized that the expression of different function-related receptors on circulating monocytes might reflect the net response of the inflammatory reaction.

Methods: We performed a prospective and observational study in a tertiary pediatric cardiac center in a population of children $(n=40)$ undergoing elective cardiac surgery. Expression of receptors on the surface of monocytes was assessed before, during, and after surgical intervention.

Results: Early monocyte activation was demonstrated by changes of the expression of the chemokine receptor CCR2, which was inversely correlated with plasma levels of monocyte chemotactic protein 1 (rho $=-0.54, P=.002$ ). High levels of monocyte chemotactic protein 1 were found in children with high expression of the adhesion receptor CD11b/CD18 on circulating monocytes. The intensity of human leukocyte antigen DR expression rapidly decreased in all children after the onset of cardiopulmonary bypass $(P<.001)$. Low human leukocyte antigen DR expression was correlated with increased plasma levels of interleukin 10 postoperatively. Children who had signs of bacterial pneumonia postoperatively had lower levels of human leukocyte antigen DR expression before surgical intervention (relative risk, $13.3 ; P=.007)$.

Conclusions: The expression of monocyte function-related receptors is altered after cardiac surgery. Early activation of monocytes by monocyte chemotactic protein 1 possibly released from the heart is followed by an anti-inflammatory response with suppression of monocyte human leukocyte antigen DR expression. The increased risk of bacterial infection after pediatric cardiac surgery can be anticipated by surveillance of monocyte function before surgical intervention.

$\mathrm{C}$ ardiac surgery with cardiopulmonary bypass (CPB) provokes the systemic inflammatory response syndrome (SIRS), which is a major cause of morbidity and mortality. The orchestrated recruitment of leukocytes is a fundamental characteristic of the inflammatory response. In this process monocytes are thought to play a key role by releasing a variety of proteases, cytotoxic proteins, and oxidants leading to tissue damage and by producing proinflammatory and antiinflammatory cytokines that might amplify or suppress the inflammatory reaction.

The CD14 molecule is one of the most characteristic surface antigens of the monocyte lineage. CD14 is highly expressed on the surface of monocytes and is strongly upregulated during the differentiation of monocytic precursors into monocytes. Functionally, CD14 is a receptor for the complex of lipopolysaccharide and its binding protein. ${ }^{1}$ On the basis of the intensity of CD14 expression, blood monocytes can be separated into 2 subpopulations. The majority of blood mono- 
cytes express CD14 at a high density (CD14 $\left.{ }^{\text {bright }}\right)$, whereas approximately $10 \%$ to $15 \%$ of monocytes express this antigen at a lower level (CD14 $\left.{ }^{\mathrm{dim}}\right)^{2,3}$ The expression of function-related antigens is different among these monocyte subpopulations. ${ }^{3,4}$

Monocytes serve an important function in cell-to-cell interaction as antigen-presenting cells. They bear the class II glycoproteins of the major histocompatibility complex gene complex: human leukocyte antigen (HLA) DR, HLA-DP, and HLA-DQ. HLA-DR expression on monocytes is increased by interferon $\gamma$ and granulocyte-macrophage colony-stimulating factor, whereas tumor necrosis factor $\alpha$, endotoxin, interleukin (IL) 10, and glucocorticoids downregulate HLA-DR expression. ${ }^{5-8}$ Decreased HLA-DR antigen expression on blood monocytes has been shown after severe trauma or major operations in adults correlating with the presence or the development of major infection. ${ }^{9}$ Moreover, low monocyte HLA-DR expression has been postulated to serve as a predictive parameter for clinical outcome in adults with sepsis ${ }^{10,11}$ and as a prognostic parameter in pediatric cardiac surgery. ${ }^{12}$

In addition to their major histocompatibility complex class II molecules, monocytes express multiple surface adhesion molecules, including CD11b/CD18 (Mac-1). $\mathrm{CD} 11 \mathrm{~b} / \mathrm{CD} 18$ is critical for adhesion and transendothelial migration. Expression of CD11b/CD18 on circulating neutrophils is decreased in critically ill patients with SIRS $^{13}$ and after CPB. ${ }^{14}$ However, no data about CD11b/CD18 expression on circulating monocyte subpopulations after $\mathrm{CPB}$ are available.

Monocytes have the ability to orient and move along a chemical gradient. Chemokines are important mediators of chemotactic and migratory behavior. These 8 - to $10-\mathrm{kd}$ molecules are subdivided into families on the basis of the relative position of the $\mathrm{N}$-terminal cysteine residues in the mature protein (CXC, $\mathrm{CC}, \mathrm{C}$, and $\mathrm{CX} 3 \mathrm{C}$ chemokines). The effects of chemokines on leukocytes are mediated by receptors that belong to the 7-transmembrane, G protein-coupled, cell-surface receptors. After stimulation, the receptors are rapidly internalized and subsequently recycled to the plasma membrane or degraded. Downregulation of receptors might therefore be an indicator of in vivo exposure of the leukocyte to the corresponding agonist. ${ }^{15}$ Whereas CXC chemokines, such as IL-8, act primarily on neutrophils, CC chemokines are potent activators of monocytes. Monocyte chemoattractant protein (MCP) 1, the prototype CC chemokine, is a potent monocyte chemoattractant that functions by interacting with the receptor CCR2, which is constitutively expressed on these cells.

The aim of the present prospective work was to investigate the expression of different membrane receptors on circulating monocytes with respect to monocyte function in this human in vivo model of systemic inflammation. We
TABLE 1. Patient characteristics

\begin{tabular}{lc}
\hline \multicolumn{1}{c}{ Age (mo) } & $\mathbf{1 1 ~ ( 3 - 8 3 ) *}$ \\
\hline Body weight (kg) & $7.5(3.5-24.4)$ \\
Left-to-right shunt lesions $†$ & $\mathrm{n}=16$ \\
Obstructive lesions $\ddagger$ & $\mathrm{n}=9$ \\
Cyanotic congenital heart defects§ & $\mathrm{n}=15$ \\
Cardiopulmonary bypass time (min) & $132(67-345)$ \\
Aortic crossclamp time (min) & $39(0-205)$
\end{tabular}

*Data are presented as medians and ranges. †Atrial septal defect $(n=7)$, ventricular septal defect $(n=5)$, complete atrioventricular canal $(n=4)$. $\ddagger$ Aortic stenosis $(n=7)$, pulmonary stenosis $(n=2)$. §Single ventricle ( $=3$ ), hypoplastic left heart syndrome $(n=3)$, tetralogy of Fallot $(n=4)$, double-outlet right ventricle ( $n=2)$, congenitally corrected transposition of the great arteries $(n=1)$, tricuspid atresia $(n=1)$, total anomalous pulmonary venous return $(n=1)$.

hypothesized that reduced HLA-DR expression reflects an anti-inflammatory state that might be a risk factor in the development of infectious complications. In contrast, a predominantly proinflammatory monocyte reaction might predispose the patient to increased cardiovascular impairment after CPB.

\section{Materials and Methods}

\section{Patients and Blood-sampling Protocol}

The study was approved by the local ethics committee. Informed written consent from the parents of each child was obtained. The studied population included 40 consecutive patients for scheduled cardiac surgery. Only children older than 3 months and younger than 7 years were included. Patients' data are shown in Table 1. All patients were breathing spontaneously. Preoperative levels of lactate were within the normal range $(1.1 \pm 0.6 \mathrm{mmol} / \mathrm{L})$, and there were no signs of infection (mean levels of C-reactive protein, $7.1 \pm 4.2 \mathrm{mg} / \mathrm{L})$.

All children received antibiotic prophylaxis at the start of the operation and 12 hours thereafter (cefazolin dose, $25 \mathrm{mg} / \mathrm{kg}$ ). Blood cultures were taken at the treating clinician's discretion if there was a clinical concern about sepsis or body core temperature of greater than $38.5^{\circ} \mathrm{C}$. All episodes of suspected infections were recorded, but for the calculation of the incidence of infectious complications, only 5 days postoperatively were taken into account. Pneumonia was defined as the presence of infiltrates on a chest radiograph plus purulent tracheobronchial secretions (Gram staining) together with positive microbial findings.

Arterial blood samples for immunophenotyping of cells, as well as for cytokine assays, were collected from each patient before CPB (after induction of anesthesia with midazolam, fentanyl, and pancuronium), during CPB (30 minutes after the start of CPB), and at 1 and 3 hours after the end of CPB. Measurements of cell receptors were done immediately after sample collection. Blood samples for cytokine assays were immediately centrifuged, and the plasma was stored at $-80^{\circ} \mathrm{C}$.

\section{CPB Management}

The extracorporeal circuit consisted of a roller pump, cardiotomy reservoir, tubing set, and oxygenator. The circuit was primed with 


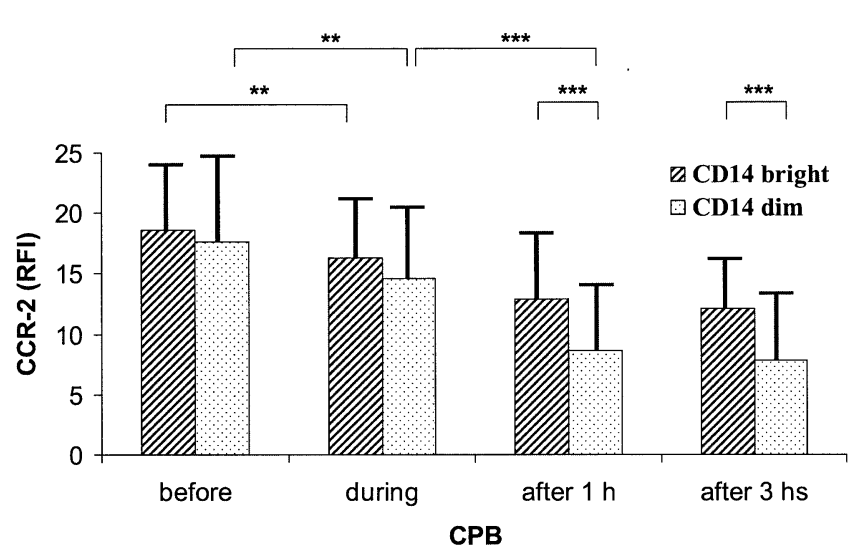

Figure 1. Expression of CCR2 (RFI) on CD14 bright (striped bars) and CD14 ${ }^{\mathrm{dim}}$ (dotted bars) monocytes during the study period. Values are expressed as means \pm standard deviation. ${ }^{* *} \boldsymbol{P}<.01$ and ${ }^{* *} \boldsymbol{P}<.001$, analysis of variance for repeated measurements and paired $t$ tests with the Bonferroni correction.

a mixture of filtered red blood cell concentrate (leukocytes $<1 \times$ 10E6 per unit), human albumin, sodium bicarbonate $8.4 \%$, mannitol 20\%, magnesium chloride, aprotinin $(250,000 \mathrm{U} / \mathrm{kg}$ body weight; Bayer, Leverkusen, Germany), and heparin (Roche Pharma, Grenzach, Switzerland). It should be pointed out that aprotinin was used in all patients, and the dosage was consistent in all patients. Patients weighing less than $7 \mathrm{~kg}$ received $30 \mathrm{mg} / \mathrm{kg}$ prednisolone (UltracortenH; Novartis Pharma, Basel, Switzerland) administered into the CPB priming solution immediately before the start of CPB according to our currently used standard treatment protocol. Cardioplegic solutions were the standard Buckberg potassium-based solutions (pharmacy of the canton of Zurich) mixed with blood. The extracorporeal circuit flow rates were between 2.4 and $2.8 \mathrm{~L} \cdot \min ^{-1} \cdot \mathrm{m}^{-2}$. Ultrafiltration was performed in all patients in a standardized manner during the rewarming period by using a blood concentrator $(20 \mathrm{~mL} / \mathrm{min}$, resulting in a volume of $800 \pm 400 \mathrm{~mL}$ ). The injected heparin was neutralized with protamine hydrochloride (ICN Pharmaceuticals, Frankfurt, Germany) after the end of CPB.

\section{Flow Cytometry of Blood Leukocytes}

Cell-surface receptors were detected with a standard technique for immunophenotyping. Arterial blood anticoagulated with ethylenediamine tetraacetic acid was used, and measurements were done immediately after sample collection. Saturating amounts of the following fluorescent monoclonal antibodies (mAbs) were used: fluorescein isothiocyanate-conjugated mouse anti-human CD14 (clone M5E2), anti-CD16-fluorescein isothiocyanate (clone 3G8), phycoerythrin (PE)-conjugated mouse anti-human CD11b/ MAC-1-PE (clone ICRF44), anti-HLA-DR-PE (clone L243) mAb, and isotype-matched controls (BD Biosciences Pharmingen, San Diego, Calif), as well as anti-human CCR2-PE (clone 48607.211) mAb (R\&D Systems, Minneapolis, Minn). Fixation of leukocytes and lysis of erythrocytes was done with FACS-lysing solution (BD Biosciences). For calibration of the measurements and for an estimation of the number of PE molecules bound per cell (PE per cell), beads conjugated with known levels of PE were used (QuantiBRITE PE, BD Biosciences). A total of 10,000 cells were acquired per measurement by using a FACSCalibur flow cytometer with CellQuest analysis software (BD Biosciences). The cells were analyzed for their fluorescence properties, and results were expressed as relative fluorescence intensity (RFI). RFI values are directly proportional to the respective expression of the receptors on the cell surface.

\section{Plasma Levels of MCP-1, IL-8, and IL-10}

Plasma concentrations of MCP-1, IL-8, and IL-10 were measured with a commercial enzyme-linked immunosorbent assay kit (Quantikine, R\&D Systems) according to the manufacturer's recommendations.

\section{Data Analysis}

Statistical analysis was performed with SPSS and GraphPad InStat 3. Unless otherwise stated, numeric results are presented as means \pm standard deviation. Two-tailed tests were used for all statistical comparisons. Data for some variables were logarithmically transformed to be approximately normally distributed. Analysis of the data at the different time points was performed with analysis of variance for repeated measurements. Paired $t$ tests with the Bonferroni correction were then performed as post-hoc tests to compare the different time points. For comparisons between monocyte subpopulations at the different time points, paired $t$ tests with the Bonferroni correction were performed. Correlations between continuous variables were assessed by using the Spearman rho correlation coefficient. Fisher exact tests have been used to compare patients with and without pneumonia with respect to dichotomized versions of HLA-DR. Mann-Whitney exact tests have been used to compare patients with and without pneumonia with respect to continuous outcomes.

\section{Results}

\section{CPB Is Followed by Diminished CCR2 Expression on Monocytes}

Separation of monocytes into 2 subsets according to their level of CD14 antigen expression, CD14 ${ }^{\text {bright }}$ and CD14 ${ }^{\mathrm{dim}}$, showed that the percentage of CD14 ${ }^{\text {bright }}$ monocytes before CPB was $90.5 \% \pm 4.3 \%$ and that this percentage remained unchanged during and after CPB (data not shown). CPB resulted in diminished CCR2 expression on both monocyte subpopulations that lasted at least 3 hours after CPB (Figure 1). The reduction of CCR2 expression was significantly more marked in $\mathrm{CD} 14^{\mathrm{dim}}$ monocytes than in $\mathrm{CD} 14^{\text {bright }}$ monocytes. The surface expression of CCR2 on CD14 ${ }^{\text {bright }}$ monocytes showed a negative correlation to the plasma levels of MCP-1 (rho $=-0.50$ and $P=.004$ at 1 hour after $\mathrm{CPB}$ and rho $=-0.54$ and $P=.002$ at 3 hours after $\mathrm{CPB}$, respectively; Figure E1). Plasma levels of MCP-1 were strongly increased during and after CPB (before CPB, 169 $\pm 152 \mathrm{pg} / \mathrm{mL}$; during CPB, $363 \pm 665 \mathrm{pg} / \mathrm{mL} ; 1$ hour after CPB, $2326 \pm 2851 \mathrm{pg} / \mathrm{mL} ; 3$ hours after CPB, $1846 \pm 2882$ $\mathrm{pg} / \mathrm{mL} ; P<.001)$. This increase was not significantly influenced by the age of the children. 


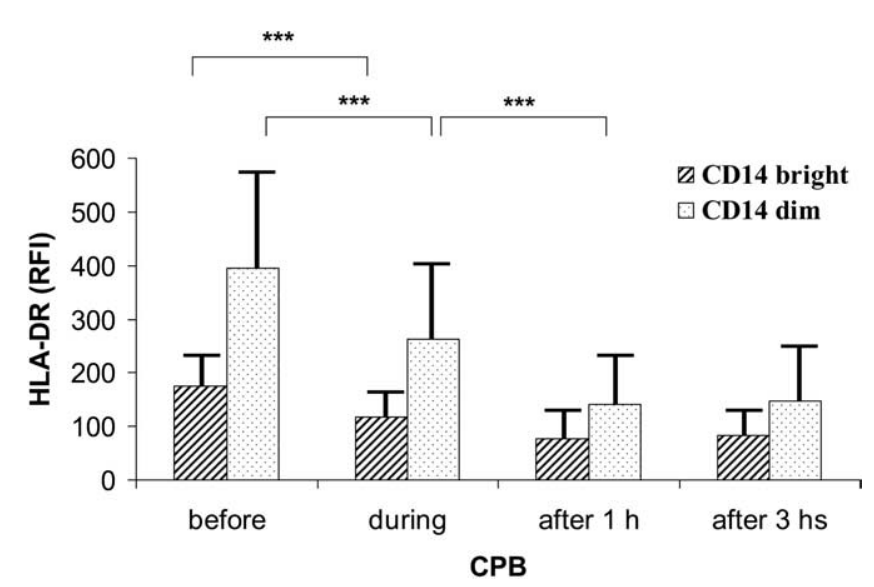

Figure 2. Expression of HLA-DR (RFI) on CD14 ${ }^{\text {bright }}$ (striped bars) and CD14 ${ }^{\text {dim }}$ (dotted bars) monocytes during the study period. Values are expressed as means \pm standard deviation. ${ }^{* * * P}<$ .001 , analysis of variance for repeated measurements and paired $t$ tests with the Bonferroni correction.

\section{Expression of Adhesion Molecule CD11b/CD18} Strongly Correlates With Plasma Levels of MCP-1 and IL-8 After CPB

$\mathrm{CD} 11 \mathrm{~b} / \mathrm{CD} 18$ is expressed on $99.9 \% \pm 0.1 \%$ of circulating monocytes. As expected, the density of CD11b/CD18 expression on $\mathrm{CD} 14^{\text {bright }}$ monocytes was higher than on CD14 ${ }^{\text {dim }}$ monocytes $(P<.001$, Figure E2). During CPB, expression of $\mathrm{CD} 11 \mathrm{~b} / \mathrm{CD} 18$ on CD14 ${ }^{\text {bright }}$ monocytes was significantly reduced. There was a significant correlation between $\mathrm{CD} 11 \mathrm{~b} / \mathrm{CD} 18$ expression on CD14 ${ }^{\text {bright }}$ monocytes and MCP-1 plasma levels at 1 and 3 hours after CPB (1 hour after $\mathrm{CPB}$, rho $=0.50$ and $P=.003 ; 3$ hours after $\mathrm{CPB}$, rho $=0.62$ and $P<.001$; Figure E3). Moreover, at 1 and 3 hours after $\mathrm{CPB}$, the density of $\mathrm{CD} 11 \mathrm{~b} / \mathrm{CD} 18$ expression on $\mathrm{CD} 14^{\text {bright }}$ monocytes was also correlated with plasma levels of IL-8 ( 1 hour after CPB, rho $=0.60$ and $P<.001 ; 3$ hours after $\mathrm{CPB}$, rho $=0.59$ and $P<.001$, respectively). Plasma levels of IL-8 were increased after CPB (1 hour after $\mathrm{CPB}, 152.0 \pm 297.3 \mathrm{pg} / \mathrm{mL} ; 3$ hours after CPB, $134.2 \pm$ $179.0 \mathrm{pg} / \mathrm{mL} ; P<.001)$ and were correlated with the duration of CPB (1 hour after $\mathrm{CPB}$, rho $=0.62$ and $P=$ $.001 ; 3$ hours after $\mathrm{CPB}$, rho $=0.51$ and $P=.004$ ). In contrast, the lowest body temperature during $\mathrm{CPB}$ and the age of the children was not significantly correlated with IL-8 levels.

The Postoperative Reduction of HLA-DR Expression on CD14 ${ }^{\text {dim }}$ Monocytes Correlates With Plasma IL-10 Levels

HLA-DR is expressed on $98.5 \% \pm 2.0 \%$ of circulating monocytes. During and after CPB, the RFI of HLA-DR expression decreased for both $\mathrm{CD} 14^{\text {bright }}$ and $\mathrm{CD} 14^{\mathrm{dim}}$

\section{(CD14 bright monocytes)}

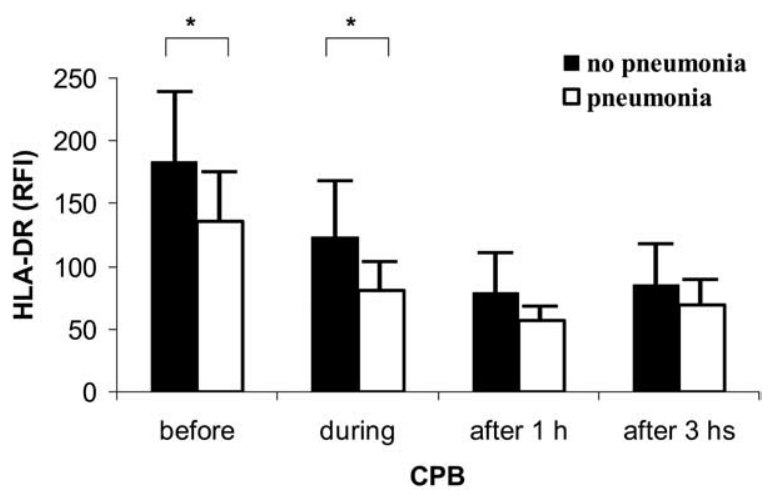

Figure 3. Expression of HLA-DR (RFI) on CD14 ${ }^{\text {bright }}$ monocytes in patients without (filled bars) and with (open bars) pneumonia during the study period. Values are expressed as means \pm standard deviation. ${ }^{*} P<.05$, analysis of variance for repeated measurements and unpaired $t$ tests with the Bonferroni correction.

monocytes (Figure 2). However, the expression of HLA-DR on $\mathrm{CD} 14^{\text {bright }}$ monocytes remained consistently much lower than on $\mathrm{CD} 14^{\mathrm{dim}}$ monocytes $(P<.01)$. The age of the children did not contribute significantly to the observed decrease of HLA-DR expression after the onset of CPB. Calculating the number of bound PE labels of HLA-DR on CD14 $4^{\text {bright }}$ monocytes, an RFI value of $176 \pm 58$ before CPB corresponds to $31,640 \pm 10,427$ PE labels per cell. In contrast, HLA-DR expression on CD14 ${ }^{\mathrm{dim}}$ monocytes before CPB was much higher (RFI value of $396 \pm 187$, corresponding to $69,306 \pm 33,499$ PE labels per cell).

Plasma levels of IL-10 were strongly increased after CPB compared with preoperative values (before CPB, 14.9 $\pm 23.2 \mathrm{pg} / \mathrm{mL}$; during CPB, $20.4 \pm 25.2 \mathrm{pg} / \mathrm{mL} ; 1$ hour after CPB, $379.2 \pm 444.3 \mathrm{pg} / \mathrm{mL} ; 3$ hours after CPB, 180.5 $\pm 197.0 \mathrm{pg} / \mathrm{mL} ; P<.001)$. No statistically significant correlation was detectable between postoperative plasma concentrations of IL-10 and the expression of HLA-DR on $\mathrm{CD} 14^{\text {bright }}$ monocytes. In contrast, there was a significant negative correlation between postoperative levels of IL-10 and the surface expression of HLA-DR on CD14 ${ }^{\text {dim }}$ monocytes (rho $=-0.35$ and $P=.032$ at 1 hour after $\mathrm{CPB}$ and rho $=-0.39$ and $P=.015$ at 3 hours after CPB; Figure E4).

Cardiac Diagnosis Did Not Significantly Affect Levels of HLA-DR Expression

The expression of HLA-DR on CD14 $4^{\text {bright }}$ monocytes in patients with or without cyanotic heart lesions was equal (HLA-DR expression in patients with cyanosis before CPB was $163 \pm 48$ RFI value, and that in patients without 
cyanosis was $184 \pm 63$ RFI value, $P=.245$; during CPB, $113 \pm 59$ vs $119 \pm 39$ RFI; 1 hour after CPB, $77 \pm 34$ vs $75 \pm 30$ RFI; 3 hours after CPB, $90 \pm 38$ vs $80 \pm 24$ RFI, respectively; $P>.30$ ). The same holds true for the expression of HLA-DR on CD14 ${ }^{\mathrm{dim}}$ monocytes, with no statistically significant differences detectable in patients with and without cyanotic heart lesions (data not shown). Low expression of HLA-DR on CD14 ${ }^{\text {bright }}$ and $\mathrm{CD} 14^{\mathrm{dim}}$ monocytes after CPB was not associated with an increased duration of postoperative inotropic therapy or increased concentrations of lactate.

\section{Patients With Pneumonia Had Significantly Lower HLA-DR Expressions Before Surgical Intervention Than Patients Without Pneumonia}

Related to infection, blood cultures were taken postoperatively from 19 patients, and 10 of these patients were treated with antibiotics. None had a positive blood culture, but tracheal mucus showed leukocytes plus positive bacterial findings in 5 patients (Staphylococcus aureus $\mathrm{n}=3$, in 2 patients together with Moraxella catarrhalis; Haemophilus influenzae $\mathrm{n}=1$, Pseudomonas aeruginosa $\mathrm{n}=1$ ). Patients with clinical and bacteriologic signs of pneumonia $(n=5)$ were younger than patients without signs of pneumonia (n $=34$; median age of 5 months [range, 3-45 months] compared with 15 months [range, 4-83 months], respectively; $P$ $=.186$ ), weighed less (median body weight of $4.5 \mathrm{~kg}$ [range, $3.5-13.2 \mathrm{~kg}$ ] compared with $10.1 \mathrm{~kg}$ [range, 4.0-24.4 $\mathrm{kg}$ ], respectively; $P=.021$ ), and were treated with steroids more often (4 of 5 patients with pneumonia compared with 19 of 34 patients without pneumonia, $P=.63$ ). One patient was excluded because of death caused by cardiac failure at 24 hours after CPB (all other patients survived until discharge). Cardiac diagnosis in both groups was quite heterogeneous, and both CPB time and aortic crossclamp time were statistically not different (data not shown). In patients with pneumonia, the expression of HLA-DR on CD14 ${ }^{\text {bright }}$ and CD14 ${ }^{\mathrm{dim}}$ monocytes was lower compared with that seen in patients without pneumonia (Figures 3 and E5). Patients with HLA-DR expression on CD14 ${ }^{\text {bright }}$ monocytes before $\mathrm{CPB}$ of less than $125 \mathrm{RFI}$ value (corresponding to 22,655 PE labels per cell) had a relative risk of 13.3 (95\% confidence interval, 1.7-104.8) of later development of pneumonia (sensitivity, 0.80; specificity, 0.85; positive predictive value, 0.44; negative predictive value, $0.97 ; P=.007)$. During CPB, a threshold of 75 RFI value (13,796 PE labels per cell) was used for calculating test statistics (sensitivity, 0.80; specificity, 0.85; positive predictive value, 0.44; negative predictive value, $0.97 ; P=.007)$. Considering HLA-DR expression on CD14 ${ }^{\mathrm{dim}}$ monocytes, only the intensity before $\mathrm{CPB}$ was useful in predicting the risk of infection (relative risk, 6.9 [95\% confidence interval, 1.433.7]; sensitivity, 0.60 ; specificity, 0.88 ; positive predictive value, 0.43 ; negative predictive value, $0.94 ; P=.032$ [threshold value used $225 \mathrm{RFI}$, corresponding to 40,086 PE labels per cell]. Low expression of HLA-DR on CD14 $4^{\text {bright }}$ and CD14 ${ }^{\mathrm{dim}}$ monocytes after CPB was not associated with an increased duration of postoperative inotropic therapy or increased concentrations of lactate.

\section{Therapy With Glucocorticoids Did Not Significantly Affect Levels of HLA-DR Expression}

Patients weighing less than $7 \mathrm{~kg}$ received glucocorticoids administered into the priming solution of the CPB circuit. Because steroids were administered into the priming solution of $\mathrm{CPB}$, the time point before $\mathrm{CPB}$ actually reflects a time when none of the patients received steroids. No significant difference was detectable between HLA-DR expression on CD14 ${ }^{\text {bright }}$ monocytes and CD14 ${ }^{\mathrm{dim}}$ monocytes at any time during the study period (HLA-DR expression on CD14 ${ }^{\text {bright }}$ monocytes in patients with steroids before CPB was $164 \pm 47$ RFI and in patients without steroids was 195 $\pm 69, P=.140$; during CPB, $125 \pm 50$ vs $104 \pm 39$ RFI; at 1 hour after $\mathrm{CPB}, 74 \pm 31$ vs $79 \pm 32$ RFI; at 3 hours after CPB, $85 \pm 36$ vs $80 \pm 15$ RFI, respectively; $P>.26$, HLA-DR expression on CD14 $4^{\text {dim }}$ monocytes, data not shown).

\section{Discussion}

This study demonstrates that cardiac surgery with CPB in children up to 7 years of age is associated with changes of the expression of surface antigens on circulating monocytes, which are correlated with changes of the monocyte function. The clinical outcome with respect to postoperative development of pneumonia was predicted by the level of HLA-DR expression before surgical intervention.

The procedure of $\mathrm{CPB}$ resulted in significant reduction of HLA-DR expression on CD14 ${ }^{\text {bright }}$ and CD14 ${ }^{\text {dim }}$ monocytes, lasting for at least 3 hours postoperatively. Decreased HLA-DR expression on circulating monocytes has been reported after major trauma or surgical intervention, as well as during the course of sepsis, reflecting monocyte deactivation. ${ }^{9-11}$ In addition, a predictive value of HLA-DR expression has been shown, with low HLA-DR expression correlating with poor outcome. ${ }^{16,17}$ Our observation of decreased HLA-DR expression after cardiac surgery confirms previous results in adults ${ }^{18}$ and children. ${ }^{12}$ In addition and noteworthy, we show that patients who experienced signs of pneumonia postoperatively had significantly lower levels of HLA-DR before the start of CPB. Only the time period of 5 days postoperatively was taken into account to assess the incidence of infections in relation to HLA-DR expression because infectious complications with nosocomial pathogens usually increase after a longer stay in the intensive care unit, irrespective of the underlying disease. Taking our data, the intensity of HLA-DR expression before CPB can be used to calculate the relative risk of postoperative development of pneumonia. 
In the present study only infants older than 3 months of age were included because reduced HLA-DR expression has been demonstrated in neonatal monocytes during the first days of life. ${ }^{3,19,20}$ By using a method that allows for the quantification of fluorescence intensity, the number of bound anti-HLA-DR mAbs expressed per monocyte before CPB was comparable with those values reported in adults. ${ }^{18}$ However, this holds true only when taking into account CD14 ${ }^{\text {bright }}$ monocytes. The majority of circulating monocytes express CD14 and HLA-DR at a high level, but about $10 \%$ of cells express CD14 at a lower level, along with increased expression of HLA-DR. ${ }^{3}$ The population of CD14 ${ }^{\text {dim }}$ monocytes with increased expression of HLA-DR has been suggested to resemble cells that have acquired features in common with more mature tissue macrophages. ${ }^{3,21}$ Moreover, the CD14 ${ }^{\text {dim }}$ HLA-DR ${ }^{\text {high }}$ monocytes show expression of a distinct pattern of cytokines compared with CD14 ${ }^{\text {bright }}$ monocytes. In particular, they have been shown in vitro to produce the proinflammatory cytokine tumor necrosis factor, whereas they produce no or little of the anti-inflammatory cytokine IL-10. ${ }^{4}$ An important finding in the present study was the significant correlation between plasma levels of IL-10 and HLA-DR expression on CD14 ${ }^{\text {dim }}$ monocytes but not between levels of IL-10 and HLA-DR expression on CD14 ${ }^{\text {bright }}$ monocytes. High levels of IL-10 have been reported in patients with sepsis and SIRS, and IL-10 has been identified as being a major monocyte deactivator. In particular, IL-10 has been demonstrated to downregulate the expression of HLA-DR on the surface of monocytes in vitro. ${ }^{7,22}$ However, in vivo no correlation between plasma levels of IL-10 and the surface expression of HLA-DR on circulating monocytes after cardiac surgery could be demonstrated. ${ }^{8}$ Our results are in agreement with findings reporting IL-10-dependent changes of HLA-DR expression according to the maturation state of monocytes. ${ }^{23-25}$ This might at least in part be responsible for conflicting results not taking into account monocyte maturation and subpopulations.

Decrease of HLA-DR expression has been shown after administration of steroids both in vitro ${ }^{26}$ and in vivo. ${ }^{27}$ In contrast, steroid treatment of patients in our study had no effect on HLA-DR expression of both monocyte subpopulations. The difference might be due to the lower dose we used or the time of administration of steroids.

A further observation of considerable interest was that after CPB, the expression of CD11b/CD18 on monocytes was correlated with plasma levels of MCP-1 and IL-8. Because CD11b/CD18 rapidly decreases on cells maintained in vitro, we used a flow cytometric assay that allows for the rapid quantification of receptor expression on the surface of cells. The expression of CD11b/CD18 was higher on $\mathrm{CD} 14^{\text {bright }}$ monocytes, confirming previously reported data. $^{3}$ The fact that one of the receptors for MCP-1, namely
CCR2, was downregulated suggests that these cells were stimulated by MCP-1. ${ }^{15}$ Indeed, we here document in vivo a rapid and sustained downregulation of CCR2 on monocytes along with increased plasma levels of MCP-1 during and after CPB in children. MCP-1 has been demonstrated in an animal model to be induced by myocardial ischemia and reperfusion. $^{28}$ Thus $\mathrm{MCP}-1$ might play a fundamental role in triggering an inflammatory reaction with recruitment and activation of monocytes after CPB in children. Activated monocytes in turn might release IL-8, as demonstrated by the correlation between CD11b/CD18 and IL-8. We have recently shown that the release of IL-8 and other proinflammatory cytokines was correlated with impairment of cardiovascular function. ${ }^{29}$

In conclusion, $\mathrm{CPB}$ in children induced rapid changes of antigen expression on the surface of circulating monocytes. The phenotypic changes are different among monocyte subpopulations, suggesting different functions. The release of MCP-1 might be an important trigger for the monocyte inflammatory response, followed by the release of humoral factors, such as IL-8 and IL-10. In cardiac surgery of children, monitoring of preoperative HLA-DR levels might be helpful in predicting an increased risk for postoperative infectious complications.

We thank the parents and their children who allowed this study to take place.

\section{References}

1. Wright SD, Ramos RA, Tobias PS, Ulevitch RJ, Mathison JC. CD14, a receptor for complexes of lipopolysaccharide (LPS) and LPS binding protein. Science. 1990;249:1431-33.

2. Passlick B, Flieger D, Ziegler-Heitbrock HWL. Identification and characterization of a novel monocyte subpopulation in human peripheral blood. Blood. 1989;74:2527-34.

3. Murphy FJ, Reen DJ. Differential expression of function-related antigens on newborn and adult monocyte subpopulations. Immunology. 1996;89:587-91

4. Belge KU, Dayyani F, Horelt A, Siedlar M, Frankenberger M, Frankenberger $\mathrm{B}$, et al. The proinflammatory CD14+CD16+DR++ monocytes are a major source of TNF. J Immunol. 2002;168:3536-42.

5. Watanabe Y, Jacob CO. Regulation of MHC class II antigen expression. Opposing effects of tumor necrosis factor- $\alpha$ on IFN- $\gamma$ induced HLA-DR and Ia expression depends on the maturation and differentiation stage of the cell. J Immunol. 1991;146:899-905.

6. Hornell TM, Beresford GW, Bushey A, Boss JM, Mellins ED. Regulation of the class II MHC pathway in primary human monocytes by granulocyte-macrophage colony-stimulating factor. J Immunol. 2003; 171:2374-83.

7. Koppelman B, Neefjes JJ, de Vries JE, de Waal-Malefyt R. Interleukin-10 down-regulates MHC class II alphabeta peptide complexes at the plasma membrane of monocytes by affecting arrival and recycling. Immunity. 1997;7:861-71.

8. Le Tulzo Y, Pangault C, Amiot L, Guilloux V, Tribut O, Arvieux C, et al. Monocyte human leukocyte antigen-DR transcriptional downregulation by cortisol during septic shock. Am J Respir Crit Care Med. 2004;169:1144-51.

9. Hershman MJ, Cheadle WG, Wellhausen SR, Davidson PF, Polk HC. Monocyte HLA-DR antigen expression characterizes clinical outcome in the trauma patient. Br J Surg. 1990;77:204-7. 
10. Saenz JJ, Izura JJ, Manrique A, Sala F, Gaminde I. Early prognosis in severe sepsis via analyzing the monocyte immunophenotype. Intensive Care Med. 2001;27:970-7.

11. Hynninen M, Pettila V, Takkunen O, Orko R, Jansson SE, Kuusela P, et al. Predictive value of monocyte histocompatibility leukocyte antigen-DR expression and plasma interleukin-4 and -10 levels in critically ill patients with sepsis. Shock. 2003;20:1-4.

12. Allen ML, Peters MJ, Goldman A, Elliott M, James I, Callard R, et al. Early postoperative monocyte deactivation predicts systemic inflammation and prolonged stay in pediatric cardiac intensive care. Crit Care Med. 2002;30:1140-5.

13. Rosenbloom AJ, Pinsky MR, Napolitano C, Nguyen TS, Levann D, Pencosky N, et al. Suppression of cytokine-mediated $\beta 2$-integrin activation on circulating neutrophils in critically ill patients. J Leukoc Biol. 1999;65:83-9.

14. Galinanes M, Watson C, Trivedi U, Chambers DJ, Young CP, Venn GE. Differential patterns of neutrophil adhesion molecules during cardiopulmonary bypass in humans. Circulation. 1996;94(suppl II): II364-9.

15. Baggiolini M. Chemokines and leukocyte traffic. Nature. 1998;392: 565-8.

16. Wakefield CH, Carey PD, Foulds S, Monson JRT, Guillou PJ. Changes in major histocompatibility complex class II expression in monocytes and T-cells in patients developing infection after surgery. Br J Surg. 1993;80:205-9.

17. Volk HD, Reinke P, Krausch D, Zuckermann H, Asadullah K, Müller $\mathrm{JM}$, et al. Monocyte deactivation-rationale for a new therapeutic strategy in sepsis. Intensive Care Med. 1996;22:474-81.

18. Oczenski W, Krenn H, Jilch R, Watzka H, Waldenberger F, Köller U, et al. HLA-DR as a marker for increased risk for systemic inflammation and septic complications after cardiac surgery. Intensive Care Med. 2003;29:1253-7.

19. Glover DM, Brownstein D, Burchett S, Larsen A, Wilson CB. Expression of HLA class II antigens and secretion of interleukin-1 by monocytes and macrophages from adults and neonates. Immunology. 1987;61:195-201.
20. Birle A, Nebe CT, Gessler P. Age-related low expression of HLA-DR molecules on monocytes of term and preterm newborns with and without signs of infection. J Perinatol. 2003;23:294-9.

21. Ziegler-Heitbrock HW, Fingerle G, Strobel M, Schraut W, Stelter F, Schutt C, et al. The novel subset of CD14+/CD16+ blood monocytes exhibits features of tissue macrophages. Eur J Immunol. 1993;23: 2053-8.

22. Fumeaux T, Pugin J. Role of interleukin-10 in the intracellular sequestration of human leukocyte antigen-DR in monocytes during septic shock. Am J Respir Crit Care Med. 2002;166:1475-82.

23. Allavena P, Piemonti L, Longoni D, Bernasconi S, Stoppacciaro A, Ruco L, et al. IL-10 prevents the differentiation of monocytes to dendritic cells but promotes their maturation to macrophages. Eur J Immunol. 1998;28:359-69.

24. Palucka KA, Taquet N, Sanchez-Chapuis F, Gluckman JC. Dendritic cells as the terminal stage of monocyte differentiation. J Immunol. 1998;160:4587-95.

25. Morel AS, Coulton G, Londei M. Regulation of major histocompatibility complex II synthesis by interleukin-10. Immunology. 2002;106: 229-36.

26. Schwiebert LM, Schleimer RP, Radka SF, Ono SJ. Modulation of MHC class II expression in human cells by dexamethasone. Cell Immunol. 1995;165:12-9.

27. Volk T, Schmutzler M, Engelhardt L, Docke WD, Volk HD, Konertz $\mathrm{W}$, et al. Influence of aminosteroid and glucocorticoid treatment on inflammation and immune function during cardiopulmonary bypass. Crit Care Med. 2001;29:2137-42.

28. Kumar AG, Ballantyne CM, Michael LH, Kukielka GL, Youker KA, Lindsey ML, et al. Induction of monocyte chemoattractant protein-1 in the small veins of the ischemic and reperfused canine myocardium. Circulation. 1997;95:693-700.

29. Gessler P, Pfenninger J, Pfammatter JP, Carrel T, Baenziger O, Dahinden C. Plasma levels of interleukin-8 and expression of interleukin-8 receptors on circulating neutrophils and monocytes after cardiopulmonary bypass in children. $J$ Thorac Cardiovasc Surg. 2003;126:718-25. 

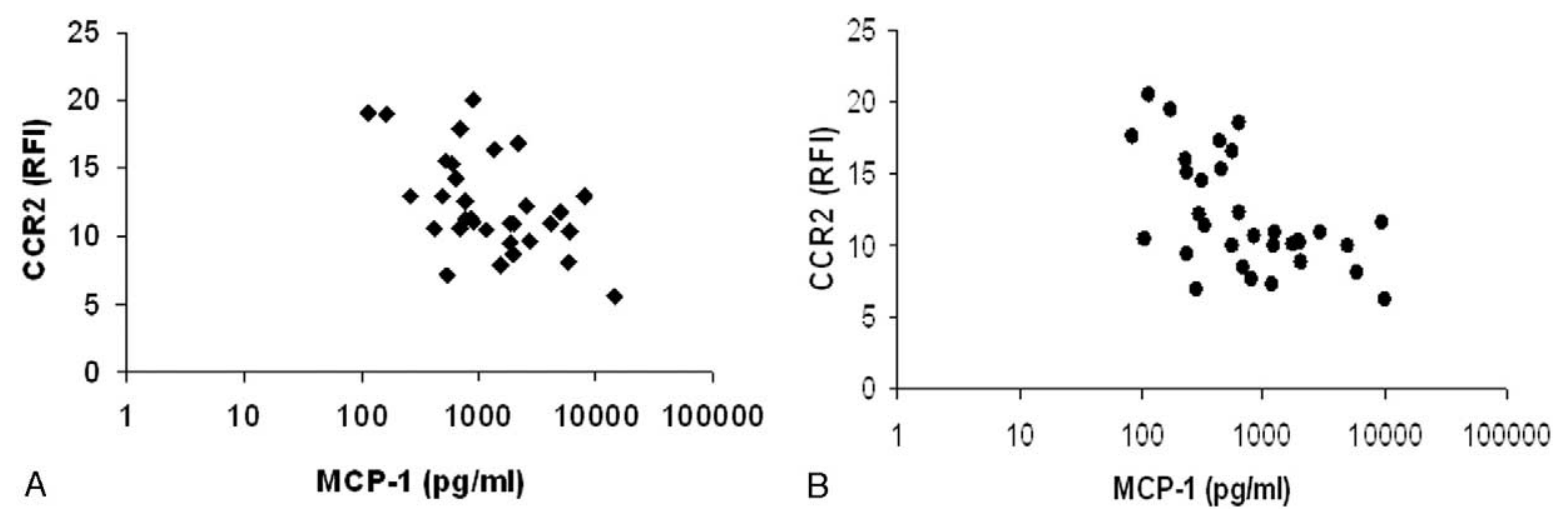

Figure E1. Correlation between the expression of CCR2 on CD14 ${ }^{\text {bright }}$ monocytes (RFI) and plasma MCP-1 concentrations (in picograms per milliliter) determined 1 hour after CPB (A) and 3 hours after CPB (B); (1 hour after CPB, rho $=-0.50$ and $P=.004 ; 3$ hours after $\mathrm{CPB}$, rho $=-0.54$ and $P=.002$ ).

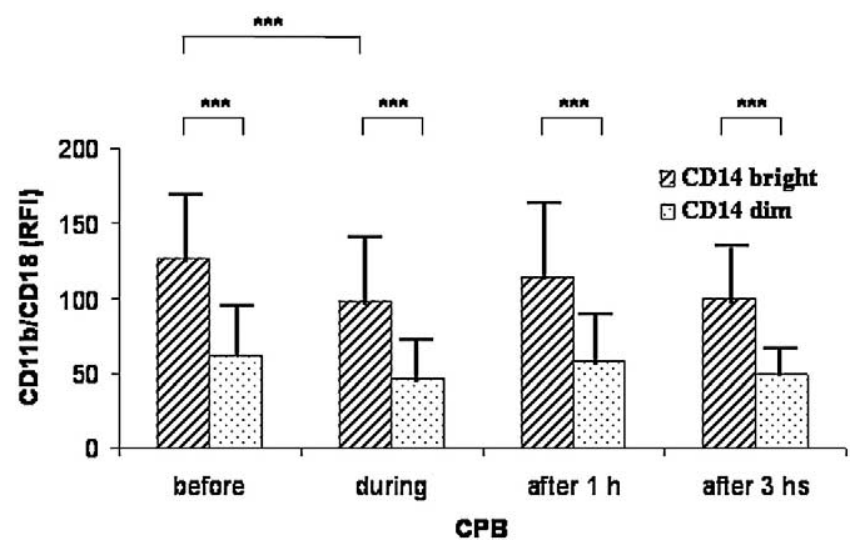

Figure E2. Expression of CD11b/CD18 (RFI) on CD14 ${ }^{\text {bright }}$ (striped bars) and CD14 ${ }^{\text {dim }}$ (dotted bars) monocytes during the study period. Values are expressed as means \pm standard deviation. ${ }^{* *} P<.001$, analysis of variance for repeated measurements and paired $t$ tests with the Bonferroni correction. 

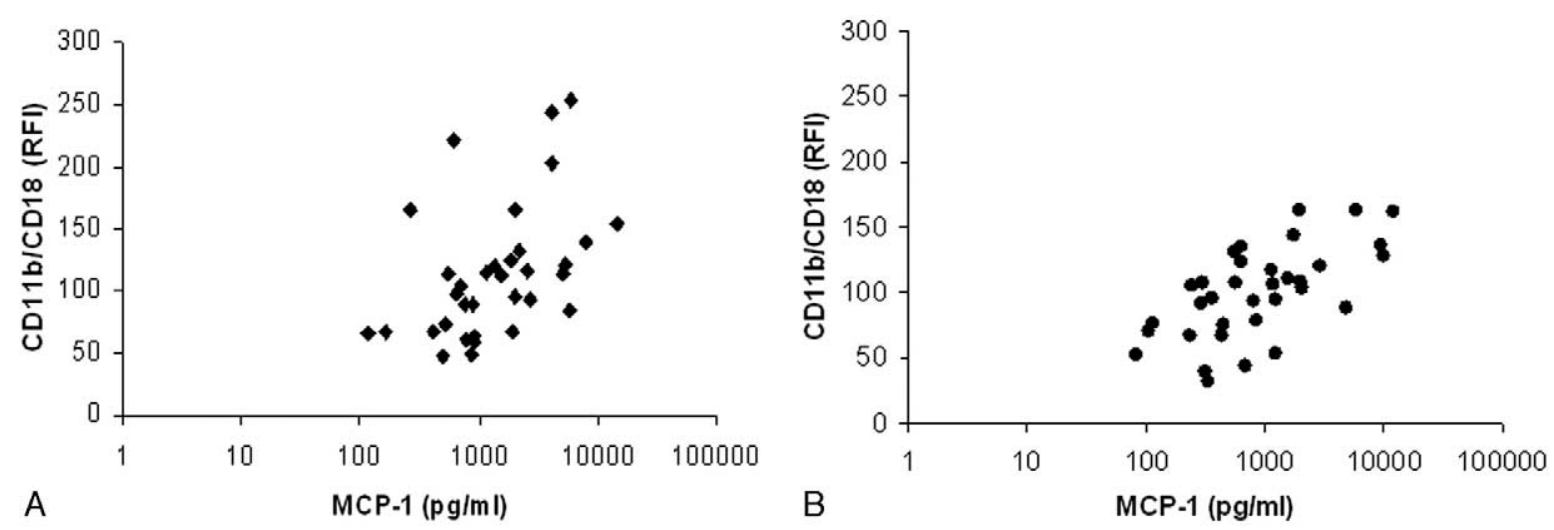

Figure E3. Correlation between the expression of CD11b/CD18 on CD14 ${ }^{\text {bright }}$ monocytes (RFI) and plasma MCP-1 concentrations (in picograms per milliliter) determined 1 hour after CPB (A) and 3 hours after CPB (B); (1 hour after CPB, rho $=0.50$ and $P=.003 ; 3$ hours after $\mathrm{CPB}$, rho $=0.62$ and $P<.001$ ).
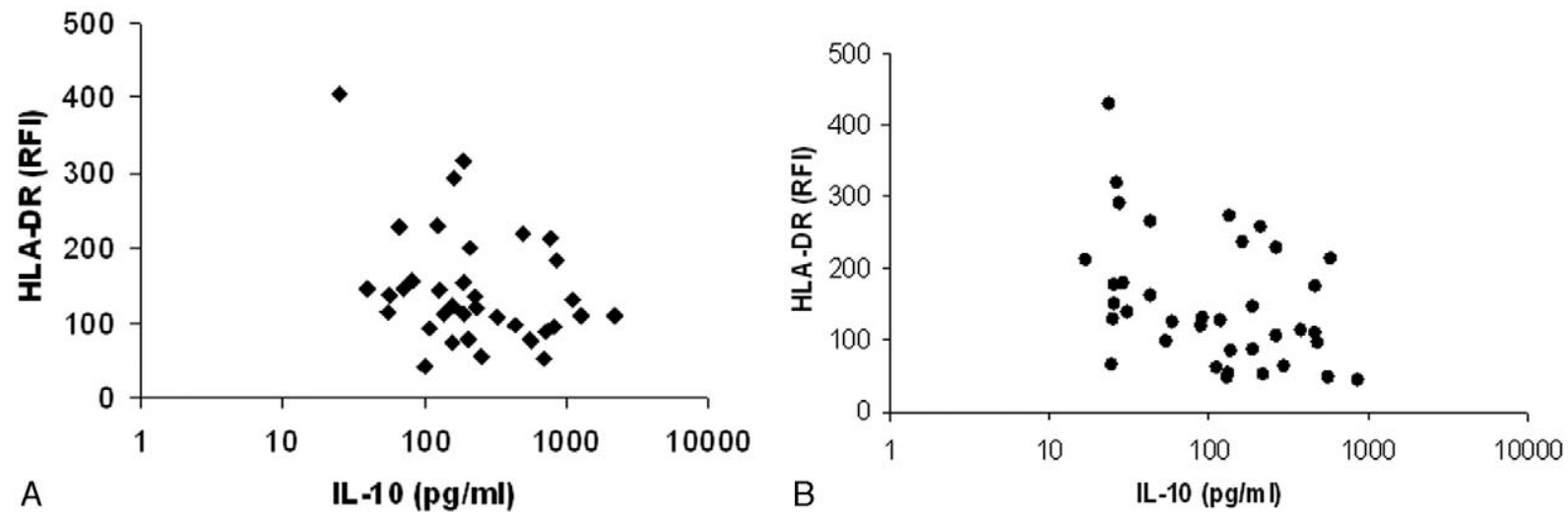

A

IL-10 (pg/ml)

B

Figure E4. Correlation between the expression of HLA-DR on CD14 ${ }^{\mathrm{dim}}$ monocytes (RFI) and plasma IL-10 concentrations (in picograms per milliliter) determined 1 hour after CPB (A) and 3 hours after CPB (B); (1 hour after CPB, rho $=-0.35$ and $P=.032 ; 3$ hours after $\mathrm{CPB}$, rho $=-0.39$ and $P=.015)$. 


\section{(CD14 dim monocytes)}

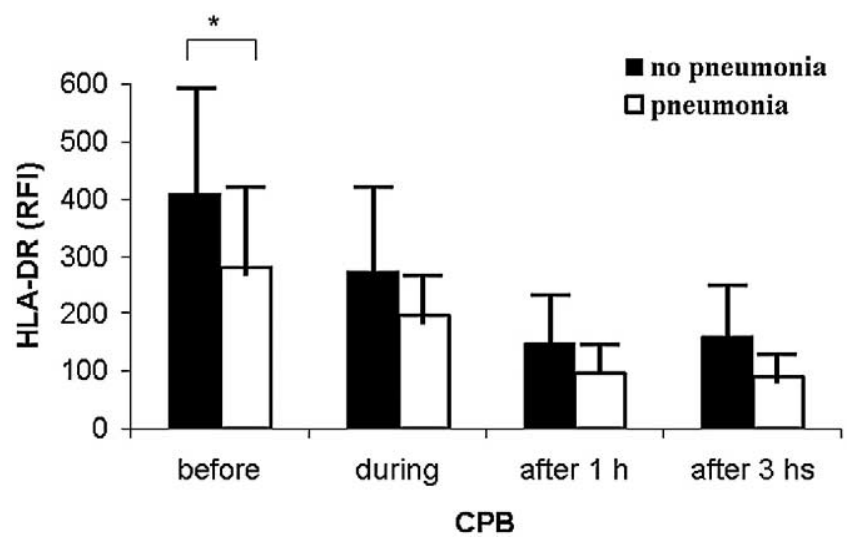

Figure E5. Expression of HLA-DR (RFI) on CD14 ${ }^{\text {dim }}$ monocytes in patients without (filled bars) and in with (open bars) pneumonia during the study period. Values are expressed as means \pm standard deviation*. $P<.05$, analysis of variance for repeated measurements and unpaired $t$ tests with the Bonferroni correction. 